\title{
Hydrofiber Dressing and Wound Repair: Review of the Literature and New Patents
}

\author{
Antonio G. Richetta ${ }^{1}$, Carmen Cantisani ${ }^{1} *$, Vincent W. Li $^{2}$, Carlo Mattozzi ${ }^{1}$, Luca Melis ${ }^{1}$, Federico \\ De Gado ${ }^{1}$, Simona Giancristoforo ${ }^{1}$ and Stefano Calvieri ${ }^{1}$
}

\author{
${ }^{I}$ Department of Dermatology and Plastic Surgery, University Sapienza of Rome, v.le del Policlinico 155, 00161; Rome, \\ Italy, ${ }^{2}$ The Angiogenesis Foundation, Cambridge, MA, Angiogenesis Center, Dermatology Department, Brigham \& \\ Women's Hospital and Harvard Medical School, Boston, MA
}

Received: May 13, 2010; Accepted: December 12, 2010; Revised: January 17, 2011

\begin{abstract}
Effective management of wound healing is a considerable challenge for clinicians. Patients underlying condition, accurate assessment of the wound and exudate, as well as selection of an appropriate dressing is all important factors for success. A variety of dressings are available to the clinician for the management of exudates. Hydrofiber dressings are a relatively new concept, and can be very cost effective because they can be worn for several days at a time. This report will review clinical evidences on the use of Hydrofiber dressing for the management of epithelial lesions, deal with current knowledge on the mechanism of action of this compound towards the epithelial wound healing process, immunological aspects and will also discuss relevant patents.
\end{abstract}

Keywords: Epithelial wound-healing, hydrofiber, inflammation, recalcitrant wounds.

\section{INTRODUCTION}

The epidermis is a barrier tissue that is exposed to the environment and susceptible to injury. Cooperation between epithelial cells, growth factors, chemokines, and inflammatory cells allows most injuries to repair, however, increasing numbers of patients suffer from chronic, non-healing wounds.

Epithelial wounds healing is a tightly regulated physiological process [1]. Under normal conditions, angiogenesis is kept in check by a balance of endogenous pro and antiangiogenic regulating factors and occurs only in a few specific circumstances for limited durations. Normal wounds can heal by secondary intention or by approximation of the wound edges, proceeding through four highly ordered distinct but overlapping phases: 1. tissue destruction, 2 . blood cell reaction, 3 . local cell reaction, 4 . remodeling of the extracellular matrix (ECM). Superficial wound healing is only supported by epithelial regeneration, while the healing of deep wounds involves ECM remodeling. Hemostasis is the result of molecular steps which ensure the formation of a plug of platelets and proteins at the site of tissue trauma (hemostatic plug) after a vascular injury, an event often occurring in epithelial wounds (primary hemostasis). Activation of the coagulation factors cascade allows fibrin polymerization which together with platelet aggregates forms a solid, stable plug. At the beginning, endothelium damage induces endothelial surface molecules which favor

\footnotetext{
*Address correspondence to this author at the Department of Dermatology and Plastic Surgery. University Sapienza of Rome, Azienda Policlinico Umberto I, Via di Boccea n. 10 int. 16, 00167 Rome Italy; Tel: +39-3479385719; Fax: +39-06490243;

E-mail: carmencantisanister@gmail.com; carmen.cantisani@uniroma1.it
}

leukocyte adhesion (i.e. selectins), while activated platelets release factors with pro-inflammatory activity. Indeed, platelets can release platelet-derived growth factor (PDGF), transforming growth factor-beta (TGF- $\beta$ ), platelet-derived epidermal growth factor (PD-EGF) platelet-derived endothelial cell growth factor (PD-ECGF), epidermal growth factor (EGF) and insulin-like growth factor-1 (IGF-1) [2]. One of the crucial steps of the process is the rapid recruitment of blood leukocytes and protein rich plasma through the vascular endothelium which accumulate in the wound. At the early stage, blood leukocytes, are mainly represented by neutrophil granulocytes. In the subsequent phase, the neutrophils flow diminishes, while the flow of monocytes predominates and differentiates into macrophages inside the wound. Both types of leukocytes are involved in cleaning the wound from debris, dead cells, degraded ECM proteins and potential pathogenic microorganisms. Both cytokines and chemokines dictate the progress of the inflammatory reaction in the wound [3]. The synthesis of the main inflammatory and immunomodulatory cytokines, including IL-1, transforming growth factor-beta (TGF- ), interferon-gamma (IFN- ), IL-4, IL-6, IL-13, and IL-17, is stimulated by tissue damage. In this context, macrophages have a key role in the switch from inflammation to wound repair [4]. The release of PDGF and TGF- by macrophages allows the recruitment of fibroblasts which increase the production of ECM proteins. Fibroblasts not only deposit ECM but are also involved in ECM remodeling. Indeed, they are an important source of matrix metallo-proteinases (MMPs), which play a key role in healing by secondary intention, and their inhibitors (TIMP), which guarantee elimination of those fibers which do not cooperate in determining the structural strength of the wound. Wound edge contraction ensures wound closure, a pivotal step lead 
by the phenotype change of fibroblasts into myofibroblasts. Concomitant with inflammation and formation of the granulation tissue, re-epithelialization is started. Reepithelialization entails: 1. migration of epithelial cells from wound borders or skin appendages, 2. proliferation of epithelial cells and 3. reestablishment of the basement membrane. The time of healing depends on systemic (age, poor nutrition, diabetes [5]) and local factors including the size of the wound, ischemia and critical infections. Indeed, acute wound healing is regarded as a process which takes place in a timely manner and in which the healing is not traumatized by complications such as infection, thrombosis or ischemia of the wound [6]. After 48 hours, uncomplicated wounds are characterized by the progression from acute inflammation to the following phases of repair. Conversely, in chronic wounds the healing phases do not occur. This latter is caused by lack of growth factors or extreme breakdown of ECM due to the unbalance between local production of proteinases and their inhibitors [7]. Indeed, the occurrence in chronic wounds of high levels of MMPs, impaired function and morphology of fibroblasts which appear insensitive to growth factors and impaired migration of keratinocytes has been demonstrated [8]. In chronic ulcers, MMP levels are markedly elevated, in contrast to their precise temporal and spatial expression in acute wounds. Both collagenase- 1 and stromelysin-1 are found in fibroblasts underlying the non-healing epithelium, and stromelysin-1 expression is especially prominent and they may play an important role in contact hypersensitivity [9].

\section{WOUND HEALING: IMMUNOLOGICAL ASPECTS}

Wound healing is a complex biological process in which the role of the immune system is not only to recognize and combat the newly presented antigens at the site of injury, but also to participate in the debridement of the damaged area and to contribute to the process of healing $[10,11]$. Skin is an active immune organ and its injury and microbial invasion trigger the activity of two important components of its innate immunity, namely epidermal dendritic cells (Langerhans cells) and antimicrobial peptides (AMPs). In humans two major families of AMPs have been characterized: defensins ( $\alpha$ and $\beta$ ) and cathelicidins. They were isolated from granules of human peripheral blood neutrophils as well as from macrophages, epithelial cells of the alimentary, respiratory and urogenital systems, and skin epidermis [12]. Other factors, such as granulocyte-macrophage colony stimulating factor (GM-CSF) and vascular endothelial growth factor (VEGF) are also critical for dendritic cell recruitment in vivo and differentiation in vitro [13]. Epidermal $\mathrm{T}$ cells have been shown to play unique roles in tissue homeostasis and repair through local secretion of distinct growth factors in the skin. Human epidermal $\mathrm{T}$ cells are able to produce insulin-like growth factor 1 (IGF-1) upon activation and promote wound healing in a skin organ culture model [14]. Identification of a human skin $\mathrm{T}$ cell equivalent with specialized wound healing properties would provide crucial insight into the mechanism of effective repair of acute wounds and elucidate new targets for therapeutic intervention in the treatment of chronic wounds. A better understanding of this complex process will lead to the development of therapeutic strategies to modulate wound repair utilizing key macrophage secretory products
[14]. Mast cells play an important role in immediate type hyper-sensitivity reactions and in the maintenance of tissue homeostasis and repair. They contain a great number of preformed mediators (heparin, histamine, tryptase, chymase, VEGF and TNF- $\alpha$ ) which affect bleeding, coagulation and acute inflammation. Their presence in older scars suggests also that they continue to be exposed to specific chemotactic, growth- and differentiation-promoting factors throughout the process of tissue remodelling. All these data indicate that mast cells contribute in a major way to wound healing. Their exact role in this process will however have to be further elucidated [15]. Antibodies might bind to wounded tissues, which would facilitate the engulfment of damaged tissues by macrophages. B cells, which produce antibodies to damaged tissues, are engaged in the wound healing process [16]. The number of tryptase-positive MCs is known to increase although the mechanical skin injury results in a reduction in $\mathrm{MC}$ counts. The reduction in $\mathrm{MC}$ counts represents a very early cytologic symptom of cell reactivity at the wound edges, even earlier than leukocytic infiltration of the wound [17]. Chronic wounds, both diabetic foot and varicous calf ulcers, showed insufficient expression of human betadefensins-2 as compared to normal skin suggesting the pathological role of this protein in chronicity of ulcers and may frequently be contaminated and colonized by bacteria [18].

\section{DRESSINGS FOR ACUTE AND CHRONIC WOUNDS}

Wounds are a major cause of morbidity (acute and chronic wounds in fact affect at least $1 \%$ of the population) and impaired quality of life therefore requires substantial health care resources in developed countries. Chronic wounds are a serious clinical problem affecting numerous elderly and diabetic patients. Regardless of etiology, wounds are difficult to treat if coexisting factors (e.g., infection or diabetes mellitus) prevent regular wound healing, therefore the choice of ideal dressing remains debatable. Wound needs to remain moist but not macerated; free of infection, toxic chemicals, particles, and fibers; at the optimum temperature and $\mathrm{pH}$ for healing; and unperturbed by the need for frequent changes [19]. Modern wound-healing concepts include different types of moist dressings and topical agents, although only a few of these treatments have convincingly been shown to give higher wound closure rates compared with traditional wet gauze dressings. A variety of topical antimicrobial agents have been developed incorporating iodine, chlorhexidine, silver, honey, topical oxygen, and topical antibiotics. Selection of appropriate strategies should be based on three key factors: heal ability (healable versus non healable), bacterial burden (colonization versus deep infection), and host risk factors. The main occlusive or semiocclusive dressings are hydrocolloid dressings (HCDs), alginates, hydrogels, foam dressings (FDs), hydrofiber dressings (HFDs), paraffin gauze and nonadherent dressings. Recent products that are reported to induce angiogenesis or reduce infection are hyaluronic acid (HA) cream or dressings and dressings supplemented with activated charcoal or silver [20]. The devices currently available substantially contain nanocomposites consisting of metal nanoparticles dispersed in dielectric matrix. Silver is the ideal metal as antibacterial agent [21]. 


\section{HYDROFIBER}

Hydrofiber (cellulose carboxymethyl fibre) dressings are a relatively new concept, and can be very cost effective because they can be worn for several days at a time. This technology is high-purity cellulose in the preformed fiber or fabric state that has been carboxymethylated in a very controlled way. It is strongly hydrophilic in nature and will rapidly absorb fluid, holding it within the structure of the fibers (a moisture retention dressing) that consists of soft non-woven sodium carboxymethylcellulose fibers. On absorbing wound fluid, the fibers transform into a clear, soft gel, while retaining overall structural integrity. The gel promotes a moist wound healing environment yet retains wound exudates by vertical absorption. Fibrin collects between the dressing and wound surface and acts as an adhesive, fixing the dressing in place and providing adherence of the dressing to the wound without in growth of tissue into the dressing. A key feature of dressings with Hydrofiber is the ability to lock in the fluid that is absorbed. This is beneficial for both caregivers and patients in terms of ease of application and removal, and reduction in pain at dressing change. This technology can be tailored to produce a whole range of wound dressings. They are therefore suitable for the management of a wide range of moderately-to-highly exuding chronic and acute wounds, such as partial thickness burns, diabetic foot/leg ulcers, pressure ulcers, traumatic wounds and surgical wounds left to heal by secondary intention [22]. This means that wound exudate, and pathogenic bacteria that it might contain, are safely removed from the wound bed and the surrounding area, protecting these surfaces from potential maceration and providing a passive mechanism of wound infection control. Furthermore, it may minimize release of bacteria into the air on dressing removal. This locking-in action, makes these dressings more effective than traditional gauze or alginate dressings at retaining fluid. In gel form, they contour closely to the wound bed avoiding "dead space" between the wound and the dressing interface, limiting spaces where bacteria can proliferate. Dealing with wound humidity, this medication, maintains a correct moisture balance of the wound bed. They are considered to have a very low allergenic potential. Nevertheless more than three percent of the treated patients developed a contact dermatitis to Hydrofiber dressing. Adverse effects included local burning and stinging on initial application, dressing hardening over joints, dressing slippage, and isolated cases of deep infection in initially infected wounds. Some particular medications add silver and its antibacterial action. Silver has been used widely for many years in wound care to help manage local infection. Various silver-impregnated wound dressings are available for the management of critically colonized and locally infected wounds for the postoperative care of surgical incisions. These dressings differ in structure and physical properties, the form and amount of silver contained in the dressing, and the mechanism by which silver is delivered. Patients treated with Ag-hydrofiber reported favourable outcome regarding ease of use, conformability, limited dressing changes, and reduced pain from dressing changes. The antimicrobial effect of silver can be explained by various mechanisms: silver interferes with the respiratory chain in the cytochromes of microbacteria; additionally, silver ions also interfere with components of the microbial electron transport system, bind DNA, and inhibit DNA replication. Little current evidence of emerging microbial resistance to silver has been reported. Reduction of matrix metalloproteinases, inhibition of pro-inflammatory cytokines, and a higher frequency of apoptosis alter inflammatory processes in the wound. Hydrofiber $\mathrm{Ag}$ dressing combines $1.2 \%$ silver to Hydrofiber dressing that is distributed throughout the dressing material. Dressings, as opposed to cream formulations, are designed to have a more controlled and prolonged release of silver during wear-time. The concept is to retain the hydrofiber's physical properties with the additional benefits of silver, which is slowly released into the wound for up to two weeks, creating a moist antimicrobial environment. The dressing entraps microorganisms within its fibers [23]. Controlled release of silver ions reduces the bioburden within the dressing, minimizing the risk of infection. The properties of Hydrofiber dressings are incorporated into a range of products formed into different textiles and formats, further enhanced chemically (e.g. through the addition of antimicrobial ionic silver), or incorporated as a specific layer into composite dressings. Hydrofiber Ag SURGICAL cover dressings are supported by evidence showing significant reduction in the incidence of superficial surgical site infection (SSI), skin blistering and delayed discharge as compared to a nonwoven post-operative surgical cover dressing regimen. Their construction provides a waterproof, viral and bacterial barrier that allows for flexibility and extensibility during body movement. With the addition of ionic silver, it also provides "on demand" broad-spectrum antimicrobial activity. Hydrofiber Ag dressings support wound healing and reduced overall pain compared with povidone-iodine gauze in the treatment of open surgical wounds [24] requiring an antimicrobial dressing killing a broad spectrum of pathogens, including methicillin-resistant Staphylococcus aureus (MRSA) [25], within 30 minutes of exposure to the dressing, for up to 7 days, as demonstrated in a simulated wound model in vitro [26]. Our experience with hydrofiber dressings was quite satisfying. We observed improvements in the wound bed associated with a reduction in patient's complaints, such as pain, after 1 or 2 applications. Five percent of our patients showed hypersensibilty to silver combined dressings diagnosed by patch testing. Therefore hydrofiber dressings containing silver should not be used on those patients sensitive to silver.

\section{CURRENT \& FUTURE DEVELOPMENTS}

Wound healing is based on the capacity of the skin to regenerate epithelia as well as connective and supporting tissue. It is marked by a complex occurrence of interweaving cell activities which drive the healing process forward. Despite major research studies, and in particular the development of hydrocolloid dressings, wound healing remains a major problem and more particularly the healing of decubitus ulcers and of deep, necrosed and exudative wounds. In fact, the management of wounds healing is frequently problematic, usually as a result of the patient's existing co-morbidities, but often because of problems in skin management, such as hyperkeratosis or skin sensitization and resulting contact dermatitis. Considering allergic contact dermatitis when a wound is recalcitrant is also 
important in the management of wounds [27]. The search for the ideal dressing is ongoing, with many new advanced dressings on the market aspiring to achieve this status [28]. No currently available dressing suits all patients or all wounds, at all stages of the healing process. The aim of new dressings is to improve the quality of care and clinical outcomes coupled with a significant reduction in the cost of providing such care. The development of wound infection is an ongoing problem for many patients. It is well documented that if a wound becomes infected, normal healing is disrupted as the inflammatory phase becomes chronic, suppressing the regenerative phase. Infected wounds may cause great distress in terms of associated morbidity and mortality, increased length of hospital admission, delayed wound healing, and increased discomfort; they also increase health care costs significantly [29]. Topical exposure of nitric oxide gas to wounds such as chronic non-healing wounds may be beneficial in promoting healing of the wound and in preparing the wound bed for further treatment and recovery [30]. New inventions include: a material with novel weave and orientation which impart low frictions qualities reducing or eliminating shear forces; in this way the potential for blister and wound formation is reduced: this will be useful for athletic individuals, neurologic, diabetic and burned patients [31]. A system and method to reduce infection rates associated with the delivery of healthcare via a series of resources to the patient [32]. A compression bandage in form of an elongated rectangle made of a textile fabric, on which a hook strip is fastened along a narrow side on a small portion of a lateral face, the hook side, the fabric containing elastic filaments running in a longitudinal direction and in the transversal directions, consists of virtually inelastic supporting filaments. The major advantage is the gradual adjustment of the compressive pressure [33]. Another new invention is a multilayer bandage for accelerating wound healing. The fibers can be formed into webbing and the webbing when brought into contact with a wound, accelerates wound healing. The webbing can be associated with all conventional dressings. The distinctive geometry and morphology of the fiber make possible a more rapid initialization and qualitative enhanced adhesion of cells to the surface of the fiber as compared to conventional bioresorbable materials such as polyglycol acid alginates and collagen enhances cell proliferation, maintenance of fiber shape and stable long term maintenance of cell metabolism [34]. Furthermore it's important to consider that any chronic wound (e.g., diabetic, decubitus, or vascular skin ulcers, complex surgical, traumatic or war wounds), can harbor numerous families of bacteria and fungi, all capable of emitting toxins. These microorganism families, each with its own profile of susceptibility or resistance to antibiotic agents, secrete different toxins, each with its own mode of noxious action. Common wound invading microorganisms include Staphylococcus, Streptococcus, Clostridium, Pseudomonas, and Coryne bacterium, among several others. A new method effective for the resolution of wounds, acute and chronic (diabetic, decubitus and vascular ulcers; surgical wounds, traumatic and war wounds), uses ozone's capacity to improve wound circulation via the activation of the nitric oxide pathway. It is able to deactivate bacterial and fungal toxins in wounds, and to disrupt wound biofilms, and related disorders. This precise device, is gently apposed to wounds, that delivers surface ozone/oxygen-via the ozone generatornot only to the biofilm's outer surface, but also to biofilms' undersurfaces where infections fester. It has minuscule hollow needles that traverse the biofilm in order to achieve this. Ozone is powerful oxidant, able to deactivate bacterial toxins, increase wound vascular activity, thus aiding cleaning of the wound site. In its gaseous form, provides superb antipathogenic action for a wide range of bacteria, viruses, protozoa, and parasites. Furthermore, ozone, in appropriately administered concentrations, due to the ongoing information about wound status possesses, has physiological properties capable of enhancing the healing of tissues. The advantages of topical ozone/oxygen therapy are: ease administration, effective antagonist to the viability of an enormous range of pathogenic organisms, appropriately applied in a timely fashion, ability to obviate the need for systemic anti-pathogen therapy, thus saving the patient from the side effects [35].

\section{CONFLICT OF INTEREST}

The authors state no conflict of interest.

\section{ACKNOWELDGEMENTS}

We would like to thank Mrs Sonia Tofani and Paolo Chilla department of dermatology and plastic surgery University "Sapienza" of Rome, Italy for their precious help.

\section{REFERENCES}

[1] Gibran NS, Boyce S, Greenhalgh DG. Cutaneous wound healing. J Burn Care Res 2007; 28(4):577-9.

[2] Kiritsy CP, Lynch AB, Lynch SE. Role of growth factors in cutaneous wound healing: A review. Crit Rev Oral Biol Med 1993; 4(5): 729-60.

[3] Manzo A, Caporali R, Montecucco C, Pitzalis C. Role of chemokines and chemokine receptors inregulating specific leukocyte trafficking in the immune/inflammatory response. Clin Exp Rheumatol 2003; 21 (4):501-8.

[4] Di Pietro LA. Wound healing: The role of the macrophage and other immune cells. Shock 1995; 4(4):233-40.

[5] Falanga V. Wound healing and its impairment in the diabetic foot. Lancet 2005; 366 (9498): 1736-43.

[6] Li J, Chen J, Kirsner R. Pathophysiology of acute wound healing. Clin Dermatol 2007; 25(1): 9-18.

[7] Toriseva M, Kähäri VM. Proteinases in cutaneous wound healing. Cell Mol Life Sci 2009; 66(2): 203-24.

[8] Braund R, Hook S, Medlicott NJ. The role of topical growth factors in chronic wounds. Curr Drug Deliv 2007; 4 (3):195-204.

[9] Pilcher BK, Wang M, Qin XJ, Parks WC, Senior RM, Welgus HG. Role of matrix metalloproteinases and their inhibition in cutaneous wound healing and allergic contact hypersensitivity. Ann N Y Acad Sci 1999; 878:12-24.

[10] Tsirogianni AK, Moutsopoulos NM, Moutsopoulos HM. Wound healing: Immunological aspects. Injury 2006; 37 (Suppl 1):S5-12.

[11] Diegelmann RF, Evans MC. Wound healing: An overview of acute, fibrotic and delayed healing. Front Biosci 2004; 9: 283-9.

[12] Oehmichen M, Kirchner H. The wound healing process. Forensic pathological aspects, Schmidt-Römhild, Lübeck, 1996;259-282.

[13] Lindner DP, Bol'shakov IN, Poberiı̌ IA, Stetsenko ON. Relationship between wound healing and the state of the immune system. Arkh Patol 1982;44(11):30-8.

[14] Toulon A, Breton L, Taylor KR, Tenenhaus M, Bhavsar D, Lanigan $\mathrm{C}$, et al. A role for human skin-resident $\mathrm{T}$ cells in wound healing. J Exp Med 2009; 206(4):743-50.

[15] Artuc M, Hermes B, Steckelings UM, Grützkau A, Henz BM. Mast cells and their mediators in cutaneous wound healing - active participants or innocent bystanders? Exp Dermatol 1999; 8(1):1-16. 
[16] Nishio N, Ito S, Suzuki H, Isobe K. Antibodies to wounded tissue enhance cutaneous wound healing. Immunology 2009; 128(3):36980.

[17] Oehmichen M, Gronki T, Meissner C, Anlauf M, Schwark T. Mast cell reactivity at the margin of human skin wounds: An early cell marker of wound survival? Forensic Sci Int 2009; 191:1-5.

[18] Galkowska H, Olszewski WL, Wojewodzka U. Expression of natural antimicrobial peptide beta-defensin- 2 and Langerhans cell accumulation in epidermis from human non-healing leg ulcers. Folia Histochem Cytobiol 2005; 43 (3):133-6.

[19] Wound Management. Available at: http://www.plymouthpct.nhs.uk/professionalzone/pajf/Documents/ pajf\%20Chapters/Ch17\%20Wound\%20Management.pdf

[20] Chaby G, Senet P, Vaneau M, Martel P, Guillaume JC, Meaume S, et al. Dressings for acute and chronic wounds: A systematic review. Arch Dermatol 2007; 143(10): 1297-304.

[21] Bellini, D., Terrassan, M.,Pavesio, A.) Biomaterials based on carboxymethylcellulose salified with zinc associated with hyaluronic acid derivatives. US20090233509 (2009).

[22] Robinson BJ. The use of a hydrofibre dressing in wound management. J Wound Care 2000; 9(1):32-34.

[23] Barnea Y, Weiss J, Gur E. A review of the applications of the hydrofiber dressing with silver (Aquacel $\mathrm{Ag}^{\circledR}$ ) in wound care. Therapeutics and Clinical Risk Management 2010:6: 21-7.

[24] Jurczak F, Dugre T, Johnstone A, Offori T, Vujovic Z, Hollander D. Randomised clinical trial of Hydrofiber dressing with silver versus povidone-iodine gauze in the management of open surgical and traumatic wounds. Int Wound J 2007; 4(1):66-76.

[25] Edwards-Jones V. Antimicrobial and barrier effects of silver against methicillin-resistant Staphylococcus aureus. J Wound Care 2006; 15(7):285-90.

[26] Poon VK, Burd A. In vitro cytotoxity of silver: Implication for clinical wound care. Burns 2004; 30(2):14-147.

[27] Siegel DM. Contact sensitivity and recalcitrant wounds. Ostomy Wound Manage 2000; 46 (1A Suppl):65S-76S.

[28] Goossens A, Cleenewerck MB. New wound dressings: Classification, tolerance. Eur J Dermatol 2010; 20(1):24-6.

[29] Lansdown AB. Silver. I: Its antibacterial properties and mechanism of action. J Wound Care. 2002 Apr;11(4):125-30.

[30] Stenzler, A., Miller, C.C. Device and method for treatment of wounds with nitric oxide. US7520866 (2009)

[31] Metsger M. B. Low friction fabric. US2008121305 (2008).

[32] Johnson, C., Akbay, K., Day, A., Doretto, G., Tu, P., Dulgeroglu, O., Peterson, M.,Toledano, D. System and method to manage delivery of healthcare to a patient. US2009089093 (2009).

[33] Naumann, D. Compression bandage. US2009143709 (2009).

[34] Thierauf, A. Baecker, I.,Haisch, A. Method for accelerated wound healing using novel fibrous webbings. US2010022932 (2010)

[35] Sunnen, G. V. Method and apparatus for the deactivation of bacterial and fungal toxins in wounds, and for the disruption of wound biofilms. US20100228183 (2010). 